\title{
Preclinical Development of Virulence Attenuated Streptococcus pneumoniae Strains Able to Enhance Protective Immunity Against
}

\section{Pneumococcal Infection}

Elisa Ramos-Sevillano, BSc, PhD ${ }^{1}$, Giuseppe Ercoli, BSc, PhD ${ }^{1}$, Philip Felgner, BSc, PhD², Rafael Ramiro de Assis, BSc, $\mathrm{PhD}^{2}$, Rie Nakajima, $\mathrm{MS}^{2}$, David Goldblatt, $\mathrm{MB}, \mathrm{ChB}, \mathrm{PhD}^{3}$, Robert $\mathrm{S}$ Heyderman, PhD, $\mathrm{FRCP}^{4}$, Stephen B Gordon, MA, MD, FRCP5 , Daniela M Ferreira, BSc, $\mathrm{PhD}^{6}$, and Jeremy S Brown, MBBS, PhD ${ }^{1 \#}$

1. Centre for Inflammation and Tissue Repair, UCL Respiratory, Division of Medicine, University College London, Rayne Institute, London WC1E 6JF, United Kingdom

2. Vaccine Research and Development Center, Department of Physiology and Biophysics, University of California Irvine, Irvine, California, USA.

3. Department of Immunobiology, UCL Great Ormond Street Institute of Child Health, NIHR Biomedical Research Centre, London, United Kingdom

4. NIHR Global Health Research Unit on Mucosal Pathogens, Research Department of Infection, Division of Infection and Immunity, University College London, Rayne Institute, London WC1E 6JF, United Kingdom

5. Malawi-Liverpool-Wellcome Trust Clinical Research Programme, Blantyre, Malawi

6. Department of Clinical Sciences, Liverpool School of Tropical Medicine, Liverpool, United Kingdom 
\#Corresponding author

Jeremy S Brown

Centre for Inflammation and Tissue Repair, Division of Medicine,

University College Medical School, Rayne Institute,

5 University Street, London WC1E 6JF, United Kingdom.

Phone: $442076796008 \quad$ Fax: 442076796973

E-mail: jeremy.brown@ucl.ac.uk

\section{Author contributions}

ERS contributed to conceiving, designing, conducting, and analyzing experiments, design of the study, and writing of the manuscript. ERS, GE, RRA, RN and PF contributed to conducting and analyzing experiments. JSB, DMF, RSH and SBG contributed to conceiving and designing the study. ERS, DG, RSH, PGA, PF contributed to designing and analyzing experiments. JSB contributed to designing, and analyzing experiments, and writing of the manuscript. All authors have read and approved the manuscript.

\section{Funding}

ER-S and GE are supported by MRC grants R/N02687X/1 and MR/R001871/1 respectively. This work was undertaken at UCLH/UCL who received a proportion of funding from the Department of Health's NIHR Biomedical Research Centre's funding scheme. RSH is supported through the National Institute for Health Research (NIHR) Global Health Research Unit on Mucosal Pathogens using UK aid from the UK Government and DG receives support from the NIHR GOS ICH Biomedical Research Centre. The views expressed in this publication are those 
of the author(s) and not necessarily those of the NIHR or the Department of Health and Social Care.

Running Title: Enhancing natural immunity using live attenuated Streptococcus pneumoniae

Descriptor number: 7.18

Key words: Streptococcus pneumoniae, colonisation, adaptive immunity, pneumonia, mucosa

Text word count: 989 


\section{To the Editors,}

The existing vaccination strategies for prevention of adult Streptococcus pneumoniae lung infections are only partially effective [1] and novel preventative approaches are required. Recent data have shown that adults develop immunity to $S$. pneumoniae through repeated episodes of asymptomatic nasopharyngeal colonisation [2-6]. This naturally acquired immunity includes protective responses to both protein and capsular antigens [2-6], and is boosted by re-colonisation events $[4,7]$. These data suggest deliberate nasopharyngeal administration of live S. pneumoniae could prevent serious S. pneumoniae infections by strengthening pre-existing cross-serotype protective immunity that inhibits nasopharyngeal colonisation with virulent strains, increases antigen-specific systemic immunity, and perhaps stengthens alveolar macrophage-mediated innate immunity $[2,3,6,7]$. This strategy would require S. pneumoniae strains able to stimulate protective immunity but unable to cause disease in a population with an underlying increased susceptibility to $S$. pneumoniae. Here we describe the development and pre-clinical characterisation of two live attenuated $S$. pneumoniae strains with these characteristics that are suitable for future use in human trials.

Fourteen mutant strains of the BHN418 6B S. pneumoniae strain containing deletions of known or potential virulence determinants (selected using data from published virulence screen and transcriptomic studies) were screened in mouse infection models for their virulence phenotypes and ability to induce protective immunity after nasopharyngeal colonisation. Eight mutations reduced virulence without affecting colonisation (data not shown). Of these, deletion of fhs or proABC (both with poorly understood roles during $S$. pneumoniae infections) caused particularly strong impairments of virulence. These genes were selected along with piaA (which encodes an iron transporter required for systemic 
virulence) as targets to make double mutant strains for investigation as candidate strains for prevention of S. pneumoniae infections. Double mutations were made to increase the degree of virulence attenuation and minimise the risk of revertant strains developing when used in human studies. Target genes were replaced from start to stop codon with the kanamycin or spectinomycin antibiotic resistance cassettes using established transformation techniques [8] to create the $\triangle$ fhs/piaA (BHN418 fhs::aad9; piaA::aphlll; $\mathrm{spc}^{\mathrm{R}} \mathrm{kan}^{\mathrm{R}}$ ) and $\triangle p r o A B C / p i a A$ (BHN418 proABC::aad9; piaA::aphIIl; $\operatorname{spc}^{R} \operatorname{kan}^{R}$ ) strains. Whole genome sequencing (MicrobesNG, Birmingham University) identified 11 and 2 non-synonmous SNPs in the $\triangle$ fhs/piaA and $\triangle p r o A B C /$ piaA strains respectively, but no other major unexpected mutations compared to the parental 6B strain (data not shown).

The $\triangle f h s / p i a A$ and $\triangle p r o A B C / p i a A$ strains were strongly reduced in systemic virulence in a mouse model of pneumonia, yet in a colonisation model maintained nasopharyngeal CFU densities similar to the wild type strain (Table 1). After two episodes of colonisation with the double mutant strains, mice developed significant serum IgG responses to the homologous BHN418 6B strain and two heterologous S. pneumoniae strains (TIGR4 and D39) (Figure 1A and B). Serum IgG responses from mice colonised with wild type, $\triangle p r o A B C / p i a A$ or $\triangle$ fhs/piaA strains were increased against the unencapsulated compared to encapsulated $6 \mathrm{~B}$ strain (Figure 1A), and when assessed using flow cytometry recognised whole wild type 6B, TIGR4 and D39 S. pneumoniae bacteria (data not shown). Together, these data suggest colonisation with the virulence-attenuated strains induced significant antibody responses mainly against non-capsular antigens. Compatible with these data, no significant anti-capsular responses were detected in serum from colonised mice using a Meso Scale Discovery (MSD) multimeric bead assay [6] (data not shown). Instead, immunoblots confirmed serum IgG from colonised 
mice recognised multiple protein bands in S. pneumoniae BHN418 6B, TIGR4 and D39 strains lysates (data not shown).

The S. pneumoniae protein antigens recognised by serum IgG from colonised mice were identified by probing a protein array containing the majority of conserved S. pneumoniae proteins recognised by naturally acquired IgG found in human sera [5]. Significant IgG responses were detected to 26 proteins (Figure 1C), with considerable overlap in the antigens recognised between mice colonised with the mutant and wild type strains. These included well-recognised immunodominant S. pneumoniae antigens (eg PsaA, PspA, SktP) as well as conserved proteins with few data on their utility as protective antigens (eg MltG, Bga, and PhtE). Importantly, subsequent pneumonia challenge in mice previously colonised with the double mutant strains was not associated with enhanced pulmonary or systemic cytokine responses (measured by MSD), or major changes in recruited inflammatory cell subsets (assessed by flow cytometry of lung preparations) compared to sham (with PBS) or $S$. pneumoniae wild type 6B colonised controls (data not shown).

When challenged using the $S$. pneumoniae BHN418 6B strain pneumonia model, mice previously colonised with the wild type BHN418 6B, $\triangle p r o A B C / p i a A$, or $\Delta f h s / p i a A$ strains were totally protected against bacteraemia (Table 1). In addition, mice colonised with the wild type or $\Delta f h s / p i a A$ strains had reduced lung CFU (Table 1). Repeat colonisation and 6B pneumonia rechallenge experiments in $\mathrm{B}$ cell deficient $\mu \mathrm{MT}$ mice or mice depleted of CD4+ cells prechallenge demonstrated an important role for antibody rather than CD4+ cells for colonisation-induced protection against septicaemia (Table 1). Previous colonisation with the $\triangle p r o A B C / p i a A$, or $\triangle f h s / p i a A$ strains also protected against recolonisation of the nasopharynx with the wild type homologous BHN418 6B strain or the heterologous TIGR4 strain, reducing 
nasal wash CFU by $>1.5 \log _{10} 7$ days after re-colonisation challenge (Table 1). If replicated in human studies this reduction in nasopharyngeal CFU is likely to impair successful nasopharyngeal colonisation by $S$. pneumoniae and thereby reduce the incidence of subsequent invasive infections. Compatible with published data showing protection against recolonisation is mediated by CD4+ effector cells targeting protein antigens $[6,9,10], C D 4+$ cell depletion abrogated the protective effect of prior colonisation against recolonisation (Table 1).

To summarise, we propose administering to the nasopharynx mutant attenuated $S$. pneumoniae strains could be a novel strategy to overcome some of the limitations of the existing vaccines. Here, we have described the design and preclinical evaluation of the $\triangle$ fhs/piaA or $\triangle p r o A B C / p i a A$ BHN418 $6 \mathrm{~B}$ mutant strains and demonstrated they are good candidate strains for testing this strategy in humans. These mutant strains can now be investigated for their protective efficacy against S. pneumoniae colonisation in a trial using an established controlled human S. pneumoniae infection challenge model [3, 7].

\section{Acknowledgements}

The 6B strain BHN418 was a gift from Prof. Birgitta Henriques Normark (Karolinska Institute). 


\section{References}

1. Jose RJ, Brown JS. Adult pneumococcal vaccination: advances, impact, and unmet needs. Curr Opin Pulm Med. 2017;23(3):225-30. Epub 2017/02/16. doi: 10.1097/MCP.0000000000000369. PubMed PMID: 28198725.

2. Ramos-Sevillano E, Ercoli G, Brown JS. Mechanisms of Naturally Acquired Immunity to Streptococcus pneumoniae. Front Immunol. 2019;10:358. Epub 2019/03/19. doi: 10.3389/fimmu.2019.00358. PubMed PMID: 30881363; PubMed Central PMCID: PMCPMC6405633.

3. Ferreira DM, Neill DR, Bangert M, Gritzfeld JF, Green N, Wright AK, et al. Controlled human infection and rechallenge with Streptococcus pneumoniae reveals the protective efficacy of carriage in healthy adults. Am J Respir Crit Care Med. 2013;187(8):855-64. Epub 2013/02/02. doi: 10.1164/rccm.201212-22770C. PubMed PMID: 23370916; PubMed Central PMCID: PMCPMC3707375.

4. Wilson R, Cohen JM, Reglinski M, Jose RJ, Chan WY, Marshall H, et al. Naturally Acquired Human Immunity to Pneumococcus Is Dependent on Antibody to Protein Antigens. PLoS pathogens. 2017;13(1):e1006137. doi: 10.1371/journal.ppat.1006137. PubMed PMID: 28135322; PubMed Central PMCID: PMC5279798.

5. Croucher NJ, Campo JJ, Le TQ, Liang X, Bentley SD, Hanage WP, et al. Diverse evolutionary patterns of pneumococcal antigens identified by pangenome-wide immunological screening. Proc Natl Acad Sci U S A. 2017;114(3):E357-E66. Epub 2017/01/06. doi: 10.1073/pnas.1613937114. PubMed PMID: 28053228; PubMed Central PMCID: PMCPMC5255586.

6. Wilson R, Cohen JM, Jose RJ, de Vogel C, Baxendale H, Brown JS. Protection against Streptococcus pneumoniae lung infection after nasopharyngeal colonization requires both humoral and cellular immune responses. Mucosal Immunol. 2015;8(3):627-39. Epub 2014/10/30. doi: 10.1038/mi.2014.95. PubMed PMID: 25354319; PubMed Central PMCID: PMCPMC4351900. 
7. Mitsi E, Carniel B, Reine J, Rylance J, Zaidi S, Soares-Schanoski A, et al. Nasal Pneumococcal Density Is Associated with Microaspiration and Heightened Human Alveolar Macrophage Responsiveness to Bacterial Pathogens. Am J Respir Crit Care Med. 2020;201(3):335-47. Epub 2019/10/19. doi: 10.1164/rccm.201903-06070C. PubMed PMID: 31626559; PubMed Central PMCID: PMCPMC6999099.

8. Khandavilli S, Homer KA, Yuste J, Basavanna S, Mitchell T, Brown JS. Maturation of Streptococcus pneumoniae lipoproteins by a type II signal peptidase is required for $A B C$ transporter function and full virulence. Molecular microbiology. 2008;67(3):541-57. doi: 10.1111/j.13652958.2007.06065.x. PubMed PMID: 18086214; PubMed Central PMCID: PMC2228790.

9. Zhang Z, Clarke TB, Weiser JN. Cellular effectors mediating Th17-dependent clearance of pneumococcal colonization in mice. J Clin Invest. 2009;119(7):1899-909. doi: 10.1172/JCI36731. PubMed PMID: 19509469; PubMed Central PMCID: PMC2701860.

10. Moffitt KL, Gierahn TM, Lu YJ, Gouveia P, Alderson M, Flechtner JB, et al. T(H)17-based vaccine design for prevention of Streptococcus pneumoniae colonization. Cell Host Microbe. 2011;9(2):15865. Epub 2011/02/16. doi: 10.1016/j.chom.2011.01.007. PubMed PMID: 21320698; PubMed Central PMCID: PMCPMC3061323. 


\section{Figure legend}

Figure 1. Serological responses in serum from mice colonised with wild type and the $\triangle$ proABC/piaA or $\triangle$ fhs/piaA S. pneumoniae strains. (A) and (B) Whole cell IgG ELISAs were performed as previously described [4, 6] using mouse sera recovered 28 days post colonisation with the wild type BHN418 6B or double mutant strains compared with uncolonised controls. (A) Whole cell ELISAs to the homologous wild type BHN418 6B (white) or unencapsulated $\triangle c p s$ BHN418 6B mutant (grey) strains (serum concentration 1 in 50). (B) Whole cell ELISAs to the heterologous TIGR4 or D39 strains (serum concentration 1 in 50). Error bars represent standard deviation and asterisks represent statistical significance between wild type and unencapsulated strains (panel A) or uncolonised controls (panel B) (Kruskal-Wallis test with Dunn's correction for multiple comparisons; ${ }^{*} p<0.05$; ${ }^{* *} p<0.01$; $\left.{ }^{* * *} p<0.001 ; * * * * p<0.0001\right)$. (C) IgG binding data to a S. pneumoniae protein antigen array probed with sera from mice colonised twice with the $6 \mathrm{~B}$ strain, $\triangle p r o A B C / p i a A$, or $\triangle f h s / p i a A$ strains. The protein array contains 289 S. pneumoniae proteins selected for their known antigenicity in humans and high degree of conservation amongst S. pneumoniae strains [5]. The array was constructed using cell free in vitro transcription/translation (IVTT) expression and printing onto nitrocellulose coated glass AVID slides (Grace Bio-Labs, Inc., Bend, OR), then probed with 1:25 mouse serum, and images acquired and analysed using an ArrayCAM $^{\circledR}$ Imaging System from Grace Bio-Labs [5]. (C) Heat map of mean IgG binding levels to the top 26 proteins recognised by IgG in colonised mouse sera ( $n=6$ mice). Results with an asterisk are statistically significantly different to the sham-colonised group (Kruskall-Wallis test to identify significant differences between groups, $p<0.05$ uncorrected for multiple comparisons). 
Table 1.

\begin{tabular}{|c|c|c|c|c|c|c|c|c|c|}
\hline \multirow[b]{2}{*}{ Infection conditions and model } & \multirow{2}{*}{$\begin{array}{l}\text { Target organ and (if } \\
\text { applicable) group }\end{array}$} & \multicolumn{2}{|c|}{ 6B wild type } & \multicolumn{2}{|l|}{$\triangle$ proABC/piaA } & \multicolumn{2}{|c|}{$\Delta$ fhs/piaA } & \multicolumn{2}{|c|}{ PBS controls } \\
\hline & & $\begin{array}{c}\log _{10} \text { CFU } \\
/ \mathrm{ml}\end{array}$ & $\mathbf{n}$ & $\begin{array}{c}\log _{10} \text { CFU } \\
/ \mathrm{ml}\end{array}$ & $\mathbf{n}$ & $\begin{array}{c}\log _{10} \text { CFU } \\
/ \mathrm{ml}\end{array}$ & $\mathbf{n}$ & $\begin{array}{c}\log _{10} \text { CFU } \\
/ \mathrm{ml}\end{array}$ & $\mathbf{n}$ \\
\hline \multicolumn{10}{|l|}{ VIRULENCE MODELS: } \\
\hline \multirow[t]{2}{*}{ Pneumonia } & Lungs & $5.26(0.55)$ & 5 & $3.78(1.50)^{\star *}$ & 5 & $4.54(0.35)$ & 5 & $\mathrm{n} / \mathrm{a}$ & \\
\hline & Blood & $3.30(0.77)$ & 5 & $0(0)^{*}$ & 5 & $0(0)^{*}$ & 5 & $\mathrm{n} / \mathrm{a}$ & \\
\hline Colonisation & Nasal wash & $4.13(0.41)$ & 10 & $3.99(0.05)$ & 5 & $3.55(0.46)$ & 5 & $\mathrm{n} / \mathrm{a}$ & \\
\hline \multicolumn{10}{|c|}{ COLONISATION THEN CHALLENGE MODELS: } \\
\hline \multirow[t]{3}{*}{ 6B pneumonia ${ }^{\dagger}$} & Lungs & $3.70(1.86)$ & 15 & $2.51(2.03)$ & $\begin{array}{l}1 \\
6\end{array}$ & $2.40(3.24)^{*}$ & 11 & $3.66(2.68)$ & 16 \\
\hline & Blood & $0(0)^{\star * *}$ & 15 & $0(0)^{* * *}$ & $\begin{array}{l}1 \\
6\end{array}$ & $0(0)^{\star * *}$ & 11 & $2.09(3.89)$ & 16 \\
\hline & BALF & $2.00(2.18)^{*}$ & 15 & $2.00(2.38)$ & $\begin{array}{l}1 \\
6 \\
\end{array}$ & $0(1.09)^{\star *}$ & 11 & $2.83(1.20)$ & 16 \\
\hline \multirow[t]{4}{*}{ 6B pneumonia $\mu \mathrm{MT}$ mice } & Lungs wild type controls & $4.54(0.86)$ & 6 & $4.10(0.50)$ & 6 & $3.11(1.46)$ & 6 & $\mathrm{n} / \mathrm{a}$ & \\
\hline & Lungs $\mu \mathrm{MT}$ mice & $4.08(0.32)$ & 3 & $4.18(0.88)$ & 6 & $4.28(0.39)$ & 6 & $\mathrm{n} / \mathrm{a}$ & \\
\hline & Blood wild type controls & $0(0)$ & 6 & $0(0)$ & 6 & $0(0)$ & 6 & $\mathrm{n} / \mathrm{a}$ & \\
\hline & Blood $\mu \mathrm{MT}$ mice & $3.54(0.77)^{*}$ & 3 & $0(2.48)$ & 6 & $3.23(0.43)^{\star *}$ & 6 & $\mathrm{n} / \mathrm{a}$ & \\
\hline \multirow[t]{4}{*}{ 6B pneumonia CD4+ depleted } & Lungs untreated controls & $3.27(2.91)$ & 6 & $3.59(2.89)$ & 6 & $3.24(2.73)$ & 6 & $\mathrm{n} / \mathrm{a}$ & \\
\hline & Lungs CD4+ depleted & $3.54(2.19)$ & 5 & $1.33(2.88)$ & 6 & $3.47(1.40)$ & 6 & $\mathrm{n} / \mathrm{a}$ & \\
\hline & Blood untreated controls & $0(0)$ & 6 & $0(0)$ & 6 & $0(0)$ & 6 & $\mathrm{n} / \mathrm{a}$ & \\
\hline & Blood CD4+ depleted & $0(0)$ & 5 & $0(0)$ & 6 & $0(0)$ & 6 & $\mathrm{n} / \mathrm{a}$ & \\
\hline 6B colonisation ${ }^{\dagger}$ & Nasal wash & $2.71(1.46)^{*}$ & 10 & $1.54(1.81)^{\star * *}$ & $\begin{array}{l}1 \\
0\end{array}$ & $2.54(0.94)^{\star *}$ & 10 & $3.88(0.16)$ & 10 \\
\hline \multirow[t]{2}{*}{ TIGR4 colonisation } & Nasal wash untreated controls & $1.00(1.78)^{\star *}$ & 5 & $1.98(2.99)^{*}$ & 6 & $1.75(1.66)^{*}$ & 6 & $3.84(0.47)$ & 6 \\
\hline & Nasal wash CD4+ depleted & $2.52(0.92)$ & 6 & $2.90(1.03)$ & 6 & $3.38(0.40)$ & 6 & $3.36(0.73)$ & 6 \\
\hline
\end{tabular}

${ }^{\dagger}$ combined data from two repeated experiments 
Table 1: In vivo phenotype analysis $\triangle p r o A B C / p i a A$ and $\triangle$ fhs/piaA mutant strains in mouse infection models. For the pneumonia model $C F U$ were obtained 28 hours post-intranasal inoculation with $1 \times 10^{7}$ S. pneumoniae CFU in $50 \mu \mathrm{l} \mathrm{PBS}$ under deep isoflurane anaesthesia, and for the colonisation model 7 days post colonisation with $1 \times 10^{7}$ CFU in $10 \mu \mathrm{PBS}$ under light isoflurane anaesthesia $[4,6,8]$. For colonisation then challenge experiments mice underwent two episodes of colonisation (day 0 and 14 ) with $1 \times 10^{7}$ CFU in $10 \mu l$ PBS under light isoflurane anaesthesia of wild type BHN418 6B, $\triangle p r o A B C / p i a A$ or $\triangle f h s / p i a A$ S. pneumoniae strains or sham-colonisation with PBS before challenge between days 30 to 42 . For these, the data presented are pooled from two experiments except colonisation with $\triangle p r o A B C / p i a A$ and then challenge experiment, which includes pooled data from three experiments. The $\mu \mathrm{MT}^{-1-}$ mice were in the $\mathrm{C} 57 \mathrm{~B} / 6 \mathrm{~J}$ background (kind gift Claudia Mauri, $\mathrm{UCL}$ ); all other experiments used CD1 mice aged 4 to 8 weeks. CD4+ cells were depleted by intraperitoneal injection of $250 \mu \mathrm{g}$ anti-CD4 mAb (GK 1.5 , BioxCell) 48 and $24 \mathrm{hrs}$ prior to S. pneumoniae challenge [6]; flow cytometry confirmed $>99 \%$ depletion of splenic CD4+ cells (data not shown). Data are presented as median of $\log _{10} \mathrm{CFU} / \mathrm{ml}$ (IQR) recovered from target organs after infection with wild type, $\triangle$ proABC/piaA, $\triangle$ fhs/piaA or PBS controls. $P$ values $(*<0.05, * *<0.01, * * *<0.001)$ were obtained using Kruskal-Wallis tests with Dunn's post hoc test comparing groups to the wild type 6B strain (for virulence models), the PBS sham-colonised (for colonisation then challenge data). The Mann-Whitney test was used for $\mu \mathrm{MT}^{-/}$mice and $\mathrm{CD} 4+$ depleted mice experiments, comparing wild type / untreated mice data to $\mu \mathrm{MT}^{-}{ }^{-} / \mathrm{CD} 4+$ depleted mice data respectively for each target organ and S. pneumoniae strain combination. Animal procedures were approved by the local ethical review process and conducted in accordance with UK national guidelines under project license PPL70/6510. 

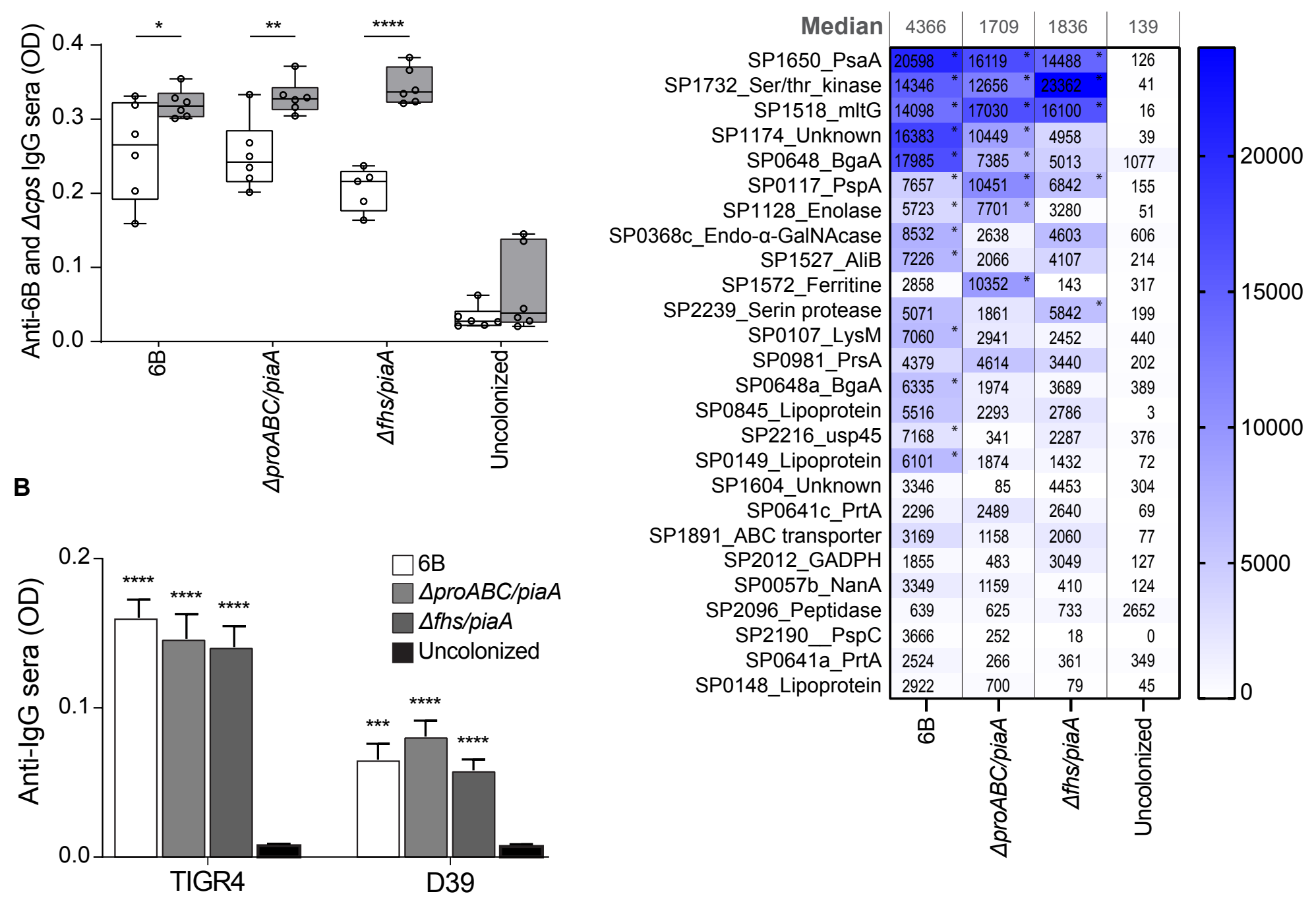

Figure 1 